\title{
Predicting mortality in critically ill patients with diabetes using machine learning and clinical notes
}

Jiancheng Ye ${ }^{1}$, Liang Yao², Jiahong Shen ${ }^{3}$, Rethavathi Janarthanam and Yuan Luo ${ }^{\text {** }}$

From The International Conference on Intelligent Biology and Medicine (ICIBM) 2020 Virtual. 9-10 August 2020

\begin{abstract}
Background: Diabetes mellitus is a prevalent metabolic disease characterized by chronic hyperglycemia. The avalanche of healthcare data is accelerating precision and personalized medicine. Artificial intelligence and algorithmbased approaches are becoming more and more vital to support clinical decision-making. These methods are able to augment health care providers by taking away some of their routine work and enabling them to focus on critical issues. However, few studies have used predictive modeling to uncover associations between comorbidities in ICU patients and diabetes. This study aimed to use Unified Medical Language System (UMLS) resources, involving machine learning and natural language processing (NLP) approaches to predict the risk of mortality.
\end{abstract}

Methods: We conducted a secondary analysis of Medical Information Mart for Intensive Care III (MIMIC-III) data. Different machine learning modeling and NLP approaches were applied. Domain knowledge in health care is built on the dictionaries created by experts who defined the clinical terminologies such as medications or clinical symptoms. This knowledge is valuable to identify information from text notes that assert a certain disease. Knowledge-guided models can automatically extract knowledge from clinical notes or biomedical literature that contains conceptual entities and relationships among these various concepts. Mortality classification was based on the combination of knowledge-guided features and rules. UMLS entity embedding and convolutional neural network (CNN) with word embeddings were applied. Concept Unique Identifiers (CUIs) with entity embeddings were utilized to build clinical text representations.

Results: The best configuration of the employed machine learning models yielded a competitive AUC of 0.97 . Machine learning models along with NLP of clinical notes are promising to assist health care providers to predict the risk of mortality of critically ill patients.

Conclusion: UMLS resources and clinical notes are powerful and important tools to predict mortality in diabetic patients in the critical care setting. The knowledge-guided $C N N$ model is effective ( $A U C=0.97$ ) for learning hidden features.

*Correspondence: yuan.luo@northwestern.edu

1 Feinberg School of Medicine, Northwestern University, Chicago, IL, USA

Full list of author information is available at the end of the article original author(s) and the source, provide a link to the Creative Commons licence, and indicate if changes were made. The images or other third party material in this article are included in the article's Creative Commons licence, unless indicated otherwise in a credit line to the material. If material is not included in the article's Creative Commons licence and your intended use is not permitted by statutory regulation or exceeds the permitted use, you will need to obtain permission directly from the copyright holder. To view a copy of this licence, visit http://creativecommons.org/licenses/by/4.0/. The Creative Commons Public Domain Dedication waiver (http://creativeco mmons.org/publicdomain/zero/1.0/) applies to the data made available in this article, unless otherwise stated in a credit line to the data. 
Keywords: ICU, Diabetic disease, Clinical notes, Machine learning, Natural language processing, Mortality, Word embedding, Entity embedding, Deep learning

\section{Background}

Diabetes mellitus is a prevalent metabolic disease characterized by chronic hyperglycemia. The rate of incidence and prevalence of patients with Diabetes mellitus type 2 among adults is increasing over time and has led to an increase in the number of patients admitted in the intensive care unit (ICU). These diabetic patients use more than $45 \%$ of resources in the ICU compared to the patients associated with other chronic diseases [1]. Additionally, it is well known that patients admitted in ICU due to diabetes are more prone to diseases and risk complication; one of these risk factors is due to the hampered immune cell response to the disease [2]. Furthermore, these risks can directly impact the survival of diabetic patients in the ICU. Only a few studies have been conducted on the mortality of diabetes mellitus patients; most of them are limited to factors associated with the increased mortality in the ICU setting [3].

The prognostic models developed previously were based on the Cox regression model and linear regression models. These models work best when the duration of diabetes is known and the data such as cohort characteristics, is a contributing factor [4]. To date, only a few studies have taken various combinations of factors into consideration to predict mortality. Anand et al. used predictive modeling, along with a combination of five key variables (type of admission, mean glucose, hemoglobin A1c, diagnoses, and age), to predict mortality, which achieved a fit with AUC values of 0.787 [3].

Meanwhile, the clinical notes that contain the patients' medical records are considered important resources to solving critical clinical issues that are difficult to obtain from other components of the electronic health records (EHR), such as laboratory data. When processed, these notes in natural language provide detailed patient information and help with clinical reasoning and inferences $[5,6]$. More recently, machine learning algorithms, natural language processing (NLP), and deep learning models have been utilized to perform text processing and classification for understanding intensive care risks. These approaches have been taking into consideration the physiological $[7,8]$, vital [9] and medication profiles [10].

Recently, text classification methods have been suggested to help in clinical document clustering; for example, some studies have utilized automated clinical document clustering for diagnosis identification and clinical procedures [11], identifying adverse drug effects [10], etc. Lexical features, such as bag-of-words or bag-of-concepts approach, are used by integrating medical ontologies, such as Unified Medical Language System (UMLS) Metathesaurus to embed clinical knowledge as machine computable information [12]. The state-of-art approach for text classification uses deep learning algorithms, such as neural network models with the distributed clinical text representation, and can learn complicated entity embeddings with the algorithms itself [11]. For instance, Yao et al. applied convolutional neural networks (CNN) with word embedding and UMLS entity embeddings to recognize and predict classes using trigger phrases [13]. Their work showed that combining domain knowledge and $\mathrm{CNN}$ models are promising for clinical text classification and outperforming obesity challenges [13]. Similarly, Hughes et al. utilized a deep learning algorithm at the sentence level for word representation with regard to medical text classification and were able to achieve a competitive model performance [14]. Domain knowledge in health care is built on the dictionaries created by experts who defined the clinical terminologies such as medications or clinical symptoms. This knowledge is valuable to identify information from text notes that assert a certain disease. Knowledge-guided models can automatically extract knowledge from clinical notes or biomedical literature that contains conceptual entities and relationships among these various concepts [15].

The avalanche of healthcare data is accelerating precision and personalized medicine. Artificial intelligence and algorithm-based approaches are becoming more and more vital to support clinical decision-making, health care delivery and health services [16-18]. These methods are able to augment health care providers by taking away some of their routine work and enabling them to focus on critical issues $[19,20]$. In this study, we proposed a new method that combines machine learning and knowledge-guided feature extraction to predict mortality among patients with diabetes mellitus. Additionally, our work demonstrates that effectively applying NLP to clinical notes and extracting meaningful features can lay the foundation for building machine learning models that are predictive for mortality in critically ill patients with diabetes. From a practical point of view, our prediction model could be used to better understand and forecast the mortality risks for critically ill patients with diabetes. 


\section{Methods}

Data were extracted from Medical Information Mart for Intensive Care-III (MIMIC-III) data using SQL queries [21]. The database contains information regarding ICU admission, medications, vitals, duration of stay, ICD9-CM diagnosis and laboratory reports. The patients with ICD-9-CM diagnosis code for diabetes mellitus (Diabetes type 1 and 2, secondary and gestational diabetes) admitted in the ICU. Pre-processing and analyses were performed using Python programming. The diabetes severity index was calculated with the points assigned for the specific ICD-9-CM codes, and the predictive model of mortality was generated with test and training sets using Python scikit-learn packages for machine learning and statistical analysis [22]. The predictive model pipeline was constructed using the clinical NLP system, clinical text classification, knowledge extraction system, the UMLS Metathesaurus, Semantic Network and learning algorithms. Multiple ICU encounters of the same patients were assigned into either a held-out test set or the training set, their information was concatenated together to form one record.

\section{Data processing}

All data processing was conducted using the Python programming language. The variables of gender, type of diabetes and severity score were calculated for each patient. The severity score was measured by the degree of organ dysfunction using the sequential organ failure assessment (SOFA) score [23]. The six organ system subscores (i.e. respiratory, coagulation, hepatic, cardiovascular, neurologic, and renal) of SOFA were scaled from 0 (no dysfunction) to 4 (severe dysfunction). The six subscores were measured in 24-h periods for the first $72 \mathrm{~h}$ of stay in all patients, and the highest score achieved was used as the clinical feature for clustering [24]. Simplified Acute Physiology Score II (SAP II) [25] and Acute Physiology Score III (APS III) [26] were calculated following the standard guidelines. These clinical information are useful to validate the performance of the models. The demographic data, clinical data and the severity score were merged into a single data frame for further analysis. The entire dataset was split in the approximate ratio of 7:3 to the training and testing sets.

\section{Clinical word and text representation}

Text classification is useful to present medical language that can be leveraged to learn the phrases that are relevant to the medical condition in the clinical notes. NLP models can extract this valuable information, in conjunction with structured data analysis, can lead to a better understanding of the diseases [27] and a more precise phenotyping of the patients [28]. The intelligent phenotyping can assist clinical decision support by improving the workflow and reviewing clinical charts, etc. The text classification was performed using phenotyping models-CNN. MetaMap [29] was applied when we identified medical concepts from clinical notes in the MIMIC-III dataset. The extracted medical concept features were from UMLS.

The UMLS Metathesaurus was used to filter clinically relevant concepts in the clinical notes [12]. To acquire UMLS concept unique identifiers (CUIs), the entity representations were used to identify and normalize lexical variants from the unstructured text content. The full clinical text was linked to CUIs in UMLS [12] via MetaMap. After entity linking, each clinical record was represented as a bag of CUIs. The UMLS CUIs were restricted within clinically relevant semantic groups and types. The neural word embedding model, word2vec, was utilized to learn word embeddings from different corpora using the continuous bag-of-words method [30].

\section{Predictive machine learning models}

The mortality rate of the patients was the primary outcome of the predictive model and we studied prediction risks of hospital mortality. The machine learning models were used to predict which diabetic patients are most likely to die in the ICU, thus providing better treatment guidance to health care providers. All model fitting was conducted using packages from Python Scikitlearn packages. The package was used to fit the regression model that contained all the relevant variables [3] to determine which variables have the greatest impact on mortality. The GLM package was used to fit the binominal logistic regression model. In the model, $70 \%$ of the sample was used as the training set, while the remaining $30 \%$ of the sample was used for validation. The key variables for these statistical machine learning predictive models include social demographics variables, such as age, gender and race, and critical clinical variables, such as hospital length of stay, SOFA scores, SAPS II, and APS III. All the feature variables are shown in Table 1; they were used in bivariate analyses to correlate with the prediction of mortality risk. The p-values less than 0.05 were considered significant for all the variables for multivariate analysis.

Following the logistic regression model, we built a random forest model to predict mortality risk using the RandomForestClassifier package with sklearn. The variables extracted from the MIMIC-III database were used in the analysis. The model was initially trained with a single decision tree, and the depth was further increased until train and test sets began to diverge. Probability estimates were used to plot the Receiver operating curve (ROC) curve. The ROC curves were generated by altering the 
Table 1 Characteristics of diabetic patients in ICU

\begin{tabular}{|c|c|c|}
\hline & $\begin{array}{l}\text { Mortality } \\
(n=1164)\end{array}$ & $\begin{array}{l}\text { Survival } \\
(\mathrm{n}=8790)\end{array}$ \\
\hline \multicolumn{3}{|l|}{ Gender, No. (\%) } \\
\hline Male & $658(56.53)$ & $5118(58.23)$ \\
\hline Female & $506(43.47)$ & $3672(41.77)$ \\
\hline Age, median (IQR), y & $73.51(63.16-80.36)$ & $66.99(57.35-76.23)$ \\
\hline \multicolumn{3}{|l|}{ Race/ethnicity, No. (\%) } \\
\hline White non-hispanic & $762(65.46)$ & $5879(66.88)$ \\
\hline Black non-hispanic & $117(10.05)$ & $1045(11.89)$ \\
\hline Hispanic & $39(3.35)$ & $382(4.35)$ \\
\hline Asian & $23(1.98)$ & $199(2.26)$ \\
\hline Other & $223(19.16)$ & $1285(14.62)$ \\
\hline Hospital LOS, median (IQR), d & $6.27(2.07-13.76)$ & $7.29(4.56-12.10)$ \\
\hline \multicolumn{3}{|l|}{ Max subscores in the first $72 \mathrm{~h}$, median (IQR) } \\
\hline Respiration & $2(0-3)$ & $0(0-2)$ \\
\hline Coagulation & $0(0-1)$ & $0(0-1)$ \\
\hline Hepatic & $0(0-1)$ & $0(0-0)$ \\
\hline Cardiovascular & $1(1-4)$ & $1(1-1)$ \\
\hline Neurologic & $3(2-4)$ & $2(0-4)$ \\
\hline Renal & $1(0-2)$ & $0(0-1)$ \\
\hline SAPS II, median (IQR) & $50(39-61)$ & $33(26-42)$ \\
\hline APS III, median (IQR) & $63.5(48-81)$ & $40(31-53)$ \\
\hline SOFA_mean in the first 7 days, median (IQR) & $6.67(4.33-9.4)$ & $3(1.71-4.67)$ \\
\hline SOFA_median in the first 7 days, median (IQR) & $7(4-10)$ & $3(1.5-5)$ \\
\hline
\end{tabular}

SOFA Sequential Organ Failure Assessment, IQR interquartile range, LOS length of stay, MODS multiple organ dysfunction syndrome, SAPS // Simplified Acute Physiology Score II, APS-III Acute Physiology Score III (APS) III

Table 2 Performance of machine learning models

\begin{tabular}{lllll}
\hline & AUC & PPV & TPR & F1 score \\
\hline Logistic regression & 0.82 & 0.63 & 0.25 & 0.35 \\
Random forest & 0.86 & 0.81 & 0.34 & 0.48 \\
AdaBoost & 0.84 & 0.68 & 0.32 & 0.44 \\
Gradient boosting & 0.83 & 0.55 & 0.39 & 0.46 \\
XGBoost & 0.87 & 0.77 & 0.37 & 0.50 \\
ANN & 0.86 & 0.77 & 0.34 & 0.47 \\
Majority voting & 0.87 & 0.82 & 0.33 & 0.47 \\
\hline
\end{tabular}

$A U C$ area under the curve, $P P V$ positive predictive value, TPR true positive rate

thresholds of the machine learning models. The performance of all the employed models were compared by area under the curve (AUC) measures. Furthermore, we evaluated more machine learning models on this diabetic cohort. (Table 2).

\section{Knowledge-guided convolutional neural networks}

To apply the Knowledge-guided CNN to clinical notes, we first identified trigger phrases using the rule that was developed to tackle semantic classification tasks [31], which were then utilized to predict classes. The trigger phrases are the name of diseases and their alternative synonyms. Next, a CNN on the trigger phrases with word embeddings and UMLS CUIs were trained. We used the Knowledge-guided CNN to combine CUI features and word features. It employed CUIs embeddings of clinical notes and pre-trained word embeddings as the input. The input layer contained word embeddings and entity embeddings of selected CUIs in each clinical record. Max pooling was utilized to select the most prominent features that have the highest values in the convolutional feature map. After that, the max pooling results of entity and word embeddings were concatenated. We adopted the same parameter settings for Knowledge-guided $\mathrm{CNN}$ from a previous study [13]; the convolution kernel size was 5, the number of convolution filters was 256, the dimension of hidden layer in the fully connected layer was 128 , dropout keep probability was 0.8 , the number of learning epochs was 30 , batch size was 64 , learning rate was 0.001 . To address imbalance, we experimented with random under-sampling with the training class ratio as $1: 3$. Under-sampling was employed to improve the classifiers to a reasonable range,;some observations in the majority class were removed [32]. 


\section{Results}

Table 1 presents characteristics of diabetic patients in the ICU. There were 9954 patients in the MIMIC-III with different types of diabetes (Diabetes type 1 and 2 , secondary and gestational diabetes). 1164 (11.69\%) of them died during the hospital course, while 8790 (88.31\%) survived. Those surviving patients had a longer hospital stay (median $=7.26$ ). We also measured the degree of organ dysfunction using the sequential organ failure assessment (SOFA) score [23] in patients admitted to the ICU. The six subscores were measured in 24-h periods for the first $72 \mathrm{~h}$ of stay in all patients, and the highest score achieved was used as the clinical feature for clustering. The 72-h time window was chosen as a proxy for the early phase of critical illness and because a large portion of organ dysfunctions tend to peak within the first days after ICU admission [33]. We also included the Simplified Acute Physiology Score (SAPS) II and Acute Physiology Score (APS) III to make the model more robust [3].

\section{Predicative machine learning models}

We ran different machine learning models to predict mortality risks using the structured EHR data. Table 2 shows the performance of various machine learning models; each model presents high sensitivity and specificity. Majority voting and XGBoost performed better than other models. Majority voting had the highest precision, while Gradient boosting had the highest recall. Both Majority voting and XGBoost had the best AUC.

Figure 1 presents the ROC for the machine learning models. When we put all the variables of interests into different models, the AUC of Majority Voting was 0.8666, which suggests that the model could predict mortality well.

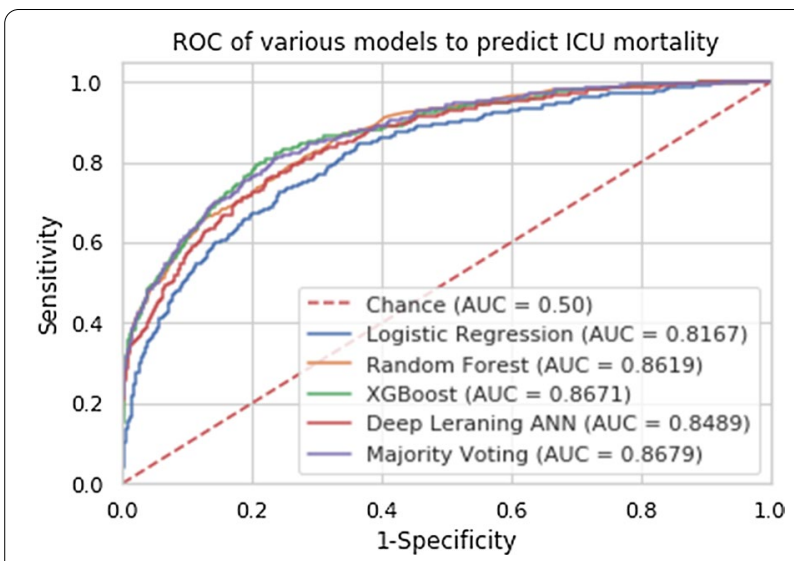

Fig. 1 Receiver operating curve (ROC) of machine learning models
Knowledge-guided convolutional neural networks

Table 3 shows the performance of CNN using word embedding and knowledge-guided CNN using $\mathrm{CUI}+$ word embedding. We note that the CNN model with word embeddings performed better than the assistant with CUIs, which means adding CUI embeddings as additional input did not improve the performance for this cohort. This is likely due to the features of diabetic diseases, as CUIs were ambiguously connected to their embeddings rather than providing more semantic information. Meanwhile, MetaMap may generate some unnecessary noise, such as irrelevant CUIs [11]. Also, some useful medical concepts may not be recognized, while some false medical concepts may be wrongly recognized when applying MetaMap.

Even so, the knowledge-guided CNN model with word embeddings still performed better than machine learning results which just utilized structured EHR data. Further studies are needed, for instance, filtering CUIs based on semantic types may improve the performance.

\section{Discussion}

Chronic diseases introduce multi-factorial issues to patients and healthcare systems, especially to critically ill patients in ICU [34]. This study contributes to different aspects that include the comparison of performance of different data representation and the supervised learning tools, such as machine learning on EHR data and NLP approaches on the medical subdomain classification using the clinical unstructured data. We also concluded that the NLP method using the UMLS concept restricted to semantic information based on the bag-of-concepts feature yielded better optimal results. The use of the standardized terminology proved to be a good knowledge representation approach, thereby leading to the possibility of future clinical EHR system integration. Likewise, the word vectors trained by our datasets may also be useful for future clinical machine learning tasks.

We also propose that our method can be used for clinical notes without medical specialization information. Identifying the clinical subdomain of a clinical note may assist clinicians in mitigating patients' unsolved problems to adequate medical specialties and experts in time. This algorithm-based method will also assist health care providers to make clinical decisions

Table 3 Performance of CNN using word embedding and knowledge-guided CNN using CUI + word embedding

\begin{tabular}{lllll}
\hline & AUC & PPV & TPR & F1 score \\
\hline CNN: text+CUls & 0.88 & 0.8915 & 0.9898 & 0.9381 \\
CNN: text & 0.97 & 0.9587 & 0.9133 & 0.9354 \\
\hline
\end{tabular}


and provide the best possible care to all the critically ill patients with diabetes.

\section{Conclusion}

In this study, we developed several predictive models to interpret the mortality of diabetes mellitus patients admitted in ICU. We observed the different performance of predictive machine learning models and their interpretability of the NLP models based on the feature sets extracted from the clinical notes. We predicted the mortality of ICU patients, taking into consideration the various factors that had statistically significant impacts on mortality. Based on the results, it is evident that the medical subdomain can be classified accurately using the clinically interpretable supervised learning based on NLP approaches.

We applied rule-based feature engineering and knowledge-guided deep learning approach to train a knowledge-guided CNN model with word embeddings and UMLS CUIs entity embeddings. The evaluation results show that the $\mathrm{CNN}$ model is effective for learning hidden features. Although CUI embeddings did not introduce improvement to the whole performance of the NLP model, they were still very helpful when building clinical text representations. More clinical databases and different patient cohorts are needed to evaluate our model in the future.

\section{Abbreviations \\ UMLS: Unified medical language system; NLP: Natural language processing; CNN: Convolutional neural network; CUI: Concept Unique Identifiers; ICU: Intensive care unit; EHR: Electronic health record; SOFA: Sequential organ failure assessment; SAP II: Simplified Acute Physiology Score II; APS III: Acute Physiology Score III; ROC: Receiver operating curve; AUC: Area under the curve; IQR: Interquartile range; LOS: Length of stay; MODS: Multiple organ dysfunc- tion syndrome; PPV: Positive predictive value; TPR: True positive rate.}

\section{Acknowledgements}

Not applicable

\section{About this supplement}

This article has been published as part of BMC Medical Informatics and Decision Making Volume 20 Supplement 11 2020: Informatics and machine learning methods for health applications. The full contents of the supplement are available at https://bmcmedinformdecismak.biomedcentral.com/articles/ supplements/volume-20-supplement-11.

\section{Authors' contributions}

J.Y. was responsible for the study design, data analysis, and initial drafting of the manuscript. L.Y. developed the KCNN system. Y.L. conceived the study, guided the study design. J.S. helped to analyze the data. R.J. helped to write the manuscript. All authors contributed to the interpretation of data for the work, the revision, and final approval of the manuscript. All authors read and approved the final manuscript.

\section{Funding}

This study is partially supported by NIH Grants R21LM012618 and R01LM013337.

\section{Availability of data and materials}

The datasets generated and/or analyzed during the current study are available on the MIMIC-III critical care database at https://mimic.physionet.org/. The relevant code and analyses are available at https://github.com/yao8839836/ obesity.

\section{Ethics approval and consent to participate}

Not applicable.

\section{Consent for publication \\ Not applicable.}

\section{Competing interests \\ None.}

\section{Author details}

${ }^{1}$ Feinberg School of Medicine, Northwestern University, Chicago, IL, USA. ${ }^{2}$ Tencent, Shenzhen, China. ${ }^{3}$ Dept. of Materials Science and Engineering, Northwestern University, Evanston, IL, USA.

Received: 6 October 2020 Accepted: 9 November 2020 Published: 30 December 2020

\section{References}

1. Burke JP, et al. Rapid rise in the incidence of type 2 diabetes from 1987 to 1996: results from the San Antonio Heart Study. Arch Intern Med. 1999;159(13):1450-6.

2. Fuchs $L$, et al. ICU admission characteristics and mortality rates among elderly and very elderly patients. Intensive Care Med. 2012;38(10):1654-61.

3. Anand RS, et al. Predicting mortality in diabetic ICU patients using machine learning and severity indices. AMIA Summits Transl Sci Proc. 2018;2018:310.

4. Chew $\mathrm{BH}$, et al. Age $\geq 60$ years was an independent risk factor for diabetes-related complications despite good control of cardiovascular risk factors in patients with type 2 diabetes mellitus. Exp Gerontol. 2013;48(5):485-91.

5. Liao KP, et al. Methods to develop an electronic medical record phenotype algorithm to compare the risk of coronary artery disease across 3 chronic disease cohorts. PLoS ONE. 2015a;10:8.

6. McCoy TH, et al. Sentiment measured in hospital discharge notes is associated with readmission and mortality risk: an electronic health record study. PLoS ONE. 2015;10:8.

7. Lin C, et al. Automatic prediction of rheumatoid arthritis disease activity from the electronic medical records. PLOS ONE. 2013;8:8.

8. Yuan J, et al. Autism spectrum disorder detection from semi-structured and unstructured medical data. EURASIP J Bioinf Syst Biol. 2016;2017(1):3.

9. Byrd RJ, et al. Automatic identification of heart failure diagnostic criteria, using text analysis of clinical notes from electronic health records. Int J Med Informatics. 2014;83(12):983-92.

10. Sarker A, Gonzalez G. Portable automatic text classification for adverse drug reaction detection via multi-corpus training. J Biomed Inform. 2015;53:196-207.

11. Weng $\mathbf{W}-\mathrm{H}$, et al. Medical subdomain classification of clinical notes using a machine learning-based natural language processing approach. BMC Med Inform Decis Mak. 2017;17(1):1-13.

12. Bodenreider $\mathrm{O}$. The unified medical language system (UMLS): integrating biomedical terminology. Nucleic Acids Res. 2004;32(suppl_1):D267-70.

13. Yao L, Mao C, Luo Y. Clinical text classification with rule-based features and knowledge-guided convolutional neural networks. BMC Med Inform Decis Mak. 2019;19(3):71.

14. Hughes M, et al. Medical text classification using convolutional neural networks. Stud Health Technol Inform. 2017;235:246-50.

15. Rindflesch TC, Fiszman M. The interaction of domain knowledge and linguistic structure in natural language processing: interpreting hypernymic propositions in biomedical text. J Biomed Inform. 2003;36(6):462-77. 
16. Zhang J, et al. A smart device for label-free and real-time detection of gene point mutations based on the high dark phase contrast of vapor condensation. Lab Chip. 2015;15(19):3891-6.

17. Li Q, et al. Label-free method using a weighted-phase algorithm to quantitate nanoscale interactions between molecules on DNA microarrays. Anal Chem. 2017;89(6):3501-7.

18. Ye J, et al. Identifying practice facilitation delays and barriers in primary care quality improvement. J Am Board Family Med. 2020;33(5):655-64.

19. Ye J. The role of health technology and informatics in a global public health emergency: practices and implications from the COVID-19 pandemic. JMIR Med Inform. 2020;8(7):e19866.

20. Ye J. Pediatric mental and behavioral health in the period of quarantine and social distancing with COVID-19. JMIR Pediatr Parent. 2020;3(2):e19867.

21. Johnson AE, et al. MIMIC-III, a freely accessible critical care database. Scientific Data. 2016;3:160035

22. Abraham A, et al. Machine learning for neuroimaging with scikit-learn. Front Neuroinform. 2014;8:14.

23. Vincent J-L, et al. The SOFA (Sepsis-related Organ Failure Assessment) score to describe organ dysfunction/failure. Berlin: Springer; 1996.

24. Ye J, Sanchez-Pinto LN. Three data-driven phenotypes of multiple organ dysfunction syndrome preserved from early childhood to middle adulthood. AMIA Annual Symposium Proceedings, 2020.

25. Le Gall J-R, Lemeshow S, Saulnier F. A new simplified acute physiology score (SAPS II) based on a European/North American multicenter study. JAMA. 1993;270(24):2957-63.

26. Pollack MM, Patel KM, Ruttimann UE. The Pediatric Risk of Mortality III-Acute Physiology Score (PRISM III-APS): a method of assessing physiologic instability for pediatric intensive care unit patients. J Pediatr. 1997;131(4):575-81.

27. Liao KP, et al. Development of phenotype algorithms using electronic medical records and incorporating natural language processing. BMJ. 2015b;350:h1885.

28. Halpern $Y$, et al. Electronic medical record phenotyping using the anchor and learn framework. J Am Med Inform Assoc. 2016;23(4):731-40.

29. Aronson AR, Lang F-M. An overview of MetaMap: historical perspective and recent advances. J Am Med Inform Assoc. 2010;17(3):229-36.

30. Luo Y, et al. Segment convolutional neural networks (Seg-CNNs) for classifying relations in clinical notes. J Am Med Inform Assoc. 2018;25(1):93-8.

31. Solt l, et al. Semantic classification of diseases in discharge summaries using a context-aware rule-based classifier. J Am Med Inform Assoc. 2009;16(4):580-4.

32. Liu $X-Y, W u$ J, Zhou Z-H. Exploratory undersampling for class-imbalance learning. IEEE Trans Syst Man Cybernet Part B (Cybernetics). 2008;2:539-50

33. Matics TJ, Sanchez-Pinto LN. Adaptation and validation of a pediatric sequential organ failure assessment score and evaluation of the sepsis-3 definitions in critically ill children. JAMA Pediatr. 2017;171(10):e172352-e172352.

34. Ye J, et al. A portable urine analyzer based on colorimetric detection. Anal Methods. 2017;9(16):2464-71.

\section{Publisher's Note}

Springer Nature remains neutral with regard to jurisdictional claims in published maps and institutional affiliations.
Ready to submit your research? Choose BMC and benefit from:

- fast, convenient online submission

- thorough peer review by experienced researchers in your field

- rapid publication on acceptance

- support for research data, including large and complex data types

- gold Open Access which fosters wider collaboration and increased citations

- maximum visibility for your research: over $100 \mathrm{M}$ website views per year

At $\mathrm{BMC}$, research is always in progress.

Learn more biomedcentral.com/submissions 